\title{
EXTRACTION OF POLYDISPERSITY INFORMATION FROM PHOTON CORRELATION DATA*
}

\author{
M. Bertero, $†$ P. Boccacci, $\dagger$ C. De Mol $\ddagger$ and E. R. Pike§ \\ †Dipartimento di Fisica dell'Università di Genova and Istituto Nazionale di Fisica Nucleare, I-16146 Genova, Italy \\ †Département de Mathématique, Université Libre de Bruxelles, B-1050 Bruxelles, Belgium \\ $\S$ Department of Physics, King's College, London WC2R 2LS, and RSRE, Great Malvern, WR 14 3PS, England
}

(Received 20 July 1987; and in final form 3 March 1988)

\begin{abstract}
Photon correlation spectroscopy (PCS) is a light-scattering technique for particle size diagnosis. It has been used mainly in the investigation of hydrosol particles since it is based on the measurement of the correlation function of the light scattered from the Brownian motion of suspended particles. Recently this technique also proved useful for studying soot particles in flames and similar aerosol systems. In the case of a polydispersed system the problem of recovering the particle size distribution can be reduced to the problem of inverting the Laplace transform. In this paper we review several methods introduced by the authors for the solution of this problem. We present some numerical results and we discuss the resolution limits characterizing the reconstruction of the size distributions.
\end{abstract}

\section{INTRODUCTION}

The method of particle sizing by laser light scattering from the Brownian motion of suspended particles has been used and refined for many years for sizing macromolecules and small particles in liquid suspension. The technique which goes under several names, for example dynamic light scattering, intensity fluctuation spectroscopy or more commonly photon correlation spectroscopy (PCS) is based upon measurement of the time dependence of the intensity of the scattered light which fluctuates due to coherent interference of the scattering from many particles in the light beam. The theory shows that the spectrum of these fluctuations is a sum of Lorenzian components, one of each particle size present. A Lorenzian spectrum for the fluctuations corresponds by the Fourier transform to an exponential photon correlation function. The decay constant of the exponential is proportional to the diffusion coefficient of the particles and the latter is proportional to the inverse of the radius of the particles, as follows from the Stokes-Einstein relation.

In most circumstances a laser beam from a stable gas laser such as helium-neon or argon-ion is directed at the particles of interest and the scattered light imaged onto a cathode of a high quality photon counting photo-multiplier tube. The intensity fluctuations in the scattered light manifest themselves in the varying rates of arrival of photoelectron impulses at the output of the detector and these are normally analysed by special high speed digital electronics in the form of a photon correlator (Cummins and Pike, 1977). In a typical experiment, $10^{7}$ or more scattered single photons will be analysed and a digital read-out of the exponential correlation function will be available with an accuracy of the order of $0.1 \%$ on each point. These points are chosen to cover a few decay times in either a linear or more preferably an integrated logarithmic sampling scheme. The data reduction problem in this monodisperse case, namely fitting a single exponential curve, poses no serious problem. When particles of different sizes, however, are present - the polydisperse case each size fraction gives rise to its own exponential curve and hence the total scattering produces an autocorrelation function which is a sum of exponentials and the data reduction problem becomes one of Laplace transform inversion.

More precisely, in the monodisperse case, the normalized modulus of the first order correlation function of the scattered electric field, such as the one measured directly in a

\footnotetext{
* Presented at the International Symposium on Optical Particle Sizing: Theory and Practice, Rouen, 12-15 May 1987.
} 
heterodyne quasi-elastic light-scattering experiment (or extracted from a homodyne experiment), is given by

$$
\left|g^{(1)}(\tau)\right|=\exp (-\Gamma \tau)
$$

where $t$ is the correlation time and

$$
\Gamma=q^{2} D, \quad D=\frac{k T}{6 \pi \eta r} .
$$

Here $q$ is the momentum transfer in the scattering experiment, $D$ the diffusion coefficient, $k$ the Boltzman constant, $T$ the temperature, $\eta$ the viscosity coefficient and $r$ the radius of the particles. The quantity $\Gamma$ is also called the characteristic linewidth associated with the particles of radius $r$. Then in the polydisperse case, the first order correlation function is given by

$$
\left|g^{(1)}(\tau)\right|=\int_{0}^{+\infty} \mathrm{e}^{-\tau \Gamma} G(\Gamma) \mathrm{d} \Gamma,
$$

where $G(\Gamma)$ is the normalized linewidth distribution function. The problem is the determination of $G(\Gamma)$ from the measured values of $\left|g^{(1)}(\tau)\right|$. As follows from equation (2), the determination of $G(\Gamma)$ is equivalent to the determination of the distribution function of the particle sizes.

Recent experiments (King et al., 1982; Chowdhury et al., 1984) have shown that there are certain applications in aerosol science where the measurement of particle size may be made by this method. In the references quoted the application was to sooting in flames. We should note that the method is only applicable to aerosols where the geometry may be chosen so that the Brownian diffusion of particles is significant compared with the other motions possible in the plane containing the incident and scattered light beams. This will normally cover the range of particles below one micrometer in diameter and therefore gives a method complimentary to particle sizing methods using the angular dependence of total scattered intensity. A departure of the data of Chowdhury et al. (1984) from a single exponential would indicate a distribution of particle sizes present in the flame. In this paper we treat the problem of estimating a particle size distribution in such an experiment and, as we have already remarked, this is equivalent to the inversion of the Laplace transform.

There is a large amount of literature on this problem but a new approach was initiated by McWhirter and Pike (1978), who introduced the eigenvalues and eigenfunctions of the Laplace transformation and were thus able to construct an 'information theoretic' inversion procedure based on the well-known Nyquist sampling ideas of standard information theory. This approach led to the consideration of a sampling scheme for a model inversion in which the reconstruction was attempted with a resolution decreasing exponentially with increase of the radius parameter (Ostrowsky et al., 1981). This is just the equivalent of the Nyquist sampling of Fourier theory when the problem is dilationally rather than translationally invariant. The corresponding method of inversion, based on simple linear least-squares procedure, is known as the 'exponential sampling method'.

As experience with this method built up it became apparent that the resolution achievable, although not high, was significantly better than that predicted by the eigenvalue spectrum. The origin of this discrepancy was shown by Bertero et al. (1982) to be due to an implicit restriction of the domain where the reconstructed solution is assumed to be different from zero: in practice finite limits must be set for numerical calculations and these, of necessity, add some a priori knowledge of the position and extent of the solution. This knowledge can be used explicitly to calculate the extra resolution possible by an extension of the method of eigenfunction expansions known as singular function expansions, well-known in pure mathematics since the last century but little used in physics until quite recently. This method is so flexible that it can also be used for the problem with discrete data and for adding several kinds of a priori information in terms of profile functions. The application of this method to Laplace transform inversion will be described in this paper. Let us stress the fact that the method applies to more general linear inverse problems when the signal detected for a monodisperse suspension is no longer a simple exponential function. In particular, our 
discussion of the limitations inherent to inversion procedures is also relevant for other problems in particle sizing such as Fraunhofer diffraction, analysis of extinction data etc.

\section{RESOLUTION LIMITS IN LAPLACE TRANSFORM INVERSION}

The problem of determining the resolution limits in Laplace inversion was solved by McWhirter and Pike (1978) using the eigenvalues and eigenfunctions of the transformation. Here we follow an equivalent approach which is based on the use of the Mellin transform. Using slightly different notations, equation (3) is a first kind Fredholm integral equation of the following form

$$
g=L f
$$

where $L$ is an integral operator given by

$$
(L f)(p)=\int_{0}^{+\infty} K(p t) f(t) \mathrm{d} t, \quad 0<p<+\infty .
$$

When $K(x)=\exp (-x)$ the integral operator $L$ is just the Laplace transformation. It is interesting to point out that a similar integral equation, with a different kernel, must also be solved in the case of the particle sizing by Fraunhofer diffraction or by extinction experiments (Bertero et al., 1987a,b). In all these applications the function $K(x)$ is real and such that $x^{-1 / 2} K(x)$ is integrable. This guarantees that the operator $L$ is bounded and selfadjoint (Reed and Simon, 1970) in the space $L^{2}(0,+\infty)$ of square-integrable functions on the semi-axis $(0,+\infty)$.

The Mellin transform of a square integrable function is defined by (Titchmarsh, 1948)

$$
\hat{f}(\omega)=\int_{0}^{+\infty} f(t) t^{-\frac{1}{2}+i \omega} \mathrm{d} t
$$

and, using well-known results (Bertero et al., 1982), the solution of equations (4) and (5) can be written in the following form

$$
f(t)=\frac{1}{2 \pi} \int_{-\infty}^{+\infty} \frac{\partial g(-\omega)}{\hat{K}(-\omega)} t^{-\left(\frac{1}{2}+i \omega\right)} \mathrm{d} \omega,
$$

where $\hat{g}(\omega)$ and $\hat{K}(\omega)$ are the Mellin transforms of $g(p)$ and $K(x)$ respectively. The inversion formula (7) however, is affected by numerical instability since $|\hat{K}(\omega)| \rightarrow 0$ when $|\omega| \rightarrow \infty$. In particular, in the case of the Laplace transform we have

$$
|\hat{K}(\omega)|^{2}=\left|\Gamma\left(\frac{1}{2}+i \omega\right)\right|^{2}=\frac{\pi}{\cosh (\pi \omega)} .
$$

In order to get stable approximate solutions one must use regularization techniques which have been developed for the treatment of ill-posed problems (Tikhonov and Arsenine, 1977). The most simple of these techniques, which is also known as optimum filtering, is the following. If we assume that the data function $g(p)$ is corrupted by additive, zero-mean, white noise with power spectrum $\varepsilon^{2}$ and that the unknown solution $f(t)$ is also from a zero-mean white noise process with power spectrum $E^{2}$, then optimum filtering consists in restricting the integral in equation (7) to the interval $\left[-\omega_{0}, \omega_{0}\right]$ where $\omega_{0}$ is the solution of the equation

$$
\left|\hat{K}\left(\omega_{0}\right)\right|^{2}=\frac{\pi}{\cosh \left(\pi \omega_{0}\right)}=\left(\frac{\varepsilon}{E}\right)^{2} .
$$

Let us denote by $\tilde{f}_{0}(t)$ the approximate solution provided by the procedure above. Then, in the case of noise free data, it is easy to show that the following relation holds true between $\tilde{f}_{0}(t)$ and the true solution $f(t)$ (Bertero et al., 1985a)

$$
\tilde{f}_{0}(t)=\int_{0}^{+\infty} M_{0}(t, s) f(s) \mathrm{d} s
$$


where

$$
M_{0}(t, s)=\frac{\sin \left[\omega_{0} \ln (t / s)\right]}{\pi(t s)^{1 / 2} \ln (t / s)} .
$$

The kernel $M_{0}(t, s)$ is the 'impulse response function' describing the total effect of both the instrument used for the measurement of the Laplace transform and the subsequent recovery procedure provided by the optimum filtering. When $f(t)=\delta(t-a)$ the recovered function is indeed $\tilde{f}(t)=M_{0}(t, a)$. The function (11) for several values of $s=a$ is plotted in Fig. 1 in the case $E / \varepsilon=10^{3}$. The typical behaviour is that the principal peak becomes broader and lower as $a$ increases. The width of the peak can be taken as a measure of the resolution achievable for the given value of the signal-to-noise ratio $E / \varepsilon$. An extension of the Rayleigh criterion, which is well-known in optics, consists of taking the position of the first zero to the right of the main peak as a resolution distance. We find a resolution distance increasing with $a$. In fact, thanks to the dilation properties of the Laplace transform, we have a constant resolution ratio $\delta_{0}$ which is defined as the ratio between the position of the zero and the value of $a$. The meaning of $\delta_{0}$ is that two exponentials of values $t_{0}, t_{1}$ cannot be resolved unless $t_{1} \geq \delta_{0} t_{0}$. From equation (11) we find that

$$
\delta_{0}=\exp \left(\pi / \omega_{0}\right) .
$$

The value of $\delta_{0}$, as a function of $E / \varepsilon$, can be easily computed using equations (9) and (12). We have $\delta_{0}=2.44,1.88,1.63$ respectively for $E / \varepsilon=10^{2}, 10^{3}, 10^{4}$.

\section{FINITE LAPLACE TRANSFORM INVERSION}

As we remarked in the Introduction it is possible to obtain an improvement of the resolution ratio defined in the previous section if we use a priori knowledge of the location and extent of the solution. This is equivalent to considering the problem of inversion of the finite Laplace transform which is defined as follows

$$
(L f)(p)=\int_{a}^{b} \mathrm{e}^{-p t} f(t) \mathrm{d} t, \quad 0<p<\infty,
$$

where $a>0$ and $b<\infty$ are given numbers. We assume in this way that the unknown function $f(t)$ vanishes outside the interval $[a, b]$ or in other words, that $f(t)$ has finite support $[a, b]$. The operator $L$ defined by equation (13) is compact from $L^{2}(a, b)$ into $L^{2}(0,+\infty)$. Let us recall that this mathematical property is important since compact operators are infinitedimensional operators with properties very close to the properties of finite matrices [for more details, we refer the reader to any standard book on basic functional analysis, for

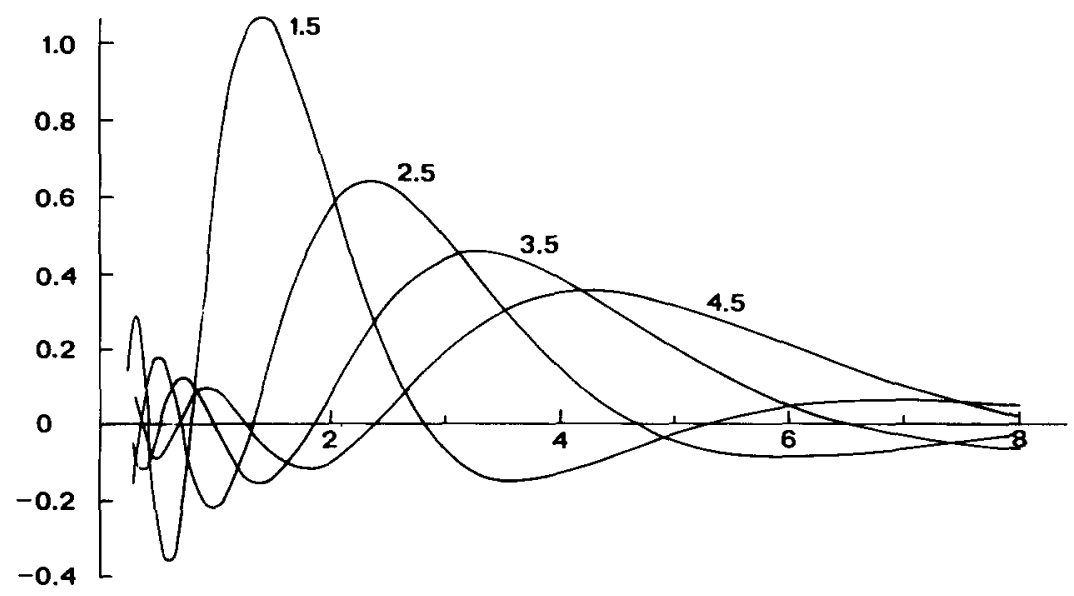

Fig. 1. Behaviour of the function $M_{0}(t, a)$ [equation (11)], for different values of $a$. The value of $\omega_{0}$ taken in these computations is 4.98 , corresponding to $E / \varepsilon=10^{3}$ in equation (6). 
example Reed and Simon (1970)]. In particular, compact operators have a discrete spectrum, so that one can introduce the so-called singular system of the operator $L$, which generalizes the concept of the eigensystem in the case of non-self-adjoint operators. The singular system of $L$ is defined as the set of the triples $\left\{\alpha_{k} ; u_{k}, v_{k}\right\}$ which solve the following coupled equations

$$
L u_{k}=\alpha_{k} v_{k}, \quad L^{*} v_{k}=\alpha_{k} u_{k},
$$

where $L^{*}$ is the adjoint operator given by

$$
\left(L^{*} g\right)(t)=\int_{0}^{+\infty} \mathrm{e}^{-t p} g(p) \mathrm{d} p .
$$

The numbers $\alpha_{k}$, real and positive by definition, are called the singular values of $L$, whereas $u_{k}$ and $v_{k}$ are called the singular functions of $L$. It is easy to show, using the dilation invariance of the Laplace transform, that the singular values $\alpha_{k}$ depend only on the ratio $\gamma=b / a$. As a consequence we can restrict ourselves to the case

$$
a=1, \quad b=\gamma .
$$

By applying the operator $L^{*}$ on both sides of the first of the equations (14) and the operator $L$ on the other equation, we see that the singular values $\alpha_{k}$ are the square roots of the eigenvalues of the self-adjoint integral operator $\tilde{L}=L^{*} L$, which is given by

$$
(\tilde{L} f)(t)=\int_{1}^{\gamma} \frac{f(s)}{t+s} \mathrm{~d} s, \quad 1 \leq t \leq \gamma
$$

and that the singular functions $u_{k}$ are the eigenfunctions of $\tilde{L}$ associated with $\alpha_{k}^{2}$. Analogously the singular functions $v_{k}$ are the eigenfunctions of the integral operator $\hat{L}=L L^{*}$

$$
(\hat{L} g)(p)=\int_{0}^{+\infty} \frac{\mathrm{e}^{-(p+q)}-\mathrm{e}^{-\gamma(p+q)}}{p+q} g(q) \mathrm{d} q
$$

associated again with the eigenvalues $\alpha_{k}^{2}$.

An important property of these singular functions, which has been proved recently (Bertero and Grünbaum, 1985), is that the singular functions $\boldsymbol{u}_{k}$ are also the eigenfunctions of the following self-adjoint, second order differential operator

$$
(\tilde{D} u)(t)=-\left[\left(t^{2}-1\right)\left(\gamma^{2}-t^{2}\right) u^{\prime}(t)\right]^{\prime}+2\left(t^{2}-1\right) u(t),
$$

the boundary conditions being provided by the requirement that $u(t)$ is bounded at $t=1$ and $t=\gamma$. Analogously the singular functions $v_{k}$ are eigenfunctions of the following fourth order differential operator

$$
(\hat{D} v)(p)=\left[p^{2} v^{\prime \prime}\right]^{\prime \prime}-\left(\gamma^{2}+1\right)\left[p^{2} v^{\prime}(p)\right]^{\prime}+\left(\gamma^{2} p^{2}-2\right) v(p) .
$$

This property is analogous to a well-known property of the prolate spheroidal wave functions of Slepian and co-workers and makes possible the accurate computation of a large number of singular functions of the finite Laplace transform (Bertero et al., 1986). Some results are plotted in Figs 2 and 3.

Since the singular functions $u_{k}(t)$ form a basis of $L^{2}(a, b)$, the unknown function can be expanded on that basis (Reed and Simon, 1970). In terms of the singular system of $L$, the inversion formula of the finite Laplace transform, i.e. the solution of the equation $g=L f$, is easily seen to be given by

$$
f(t)=\sum_{k=0}^{+\infty} \frac{1}{\alpha_{k}}\left(g, v_{k}\right) u_{k}(t)
$$

where $\left(g, v_{k}\right)$ is the usual scalar product of $L^{2}(0,+\infty)$. This formula is affected by numerical instability as equation (7), since the singular values $\alpha_{k}$ of a compact operator tend to zero when $k \rightarrow \infty$ (Reed and Simon, 1970). When $\gamma$ is small they tend to zero very rapidly indeed. 


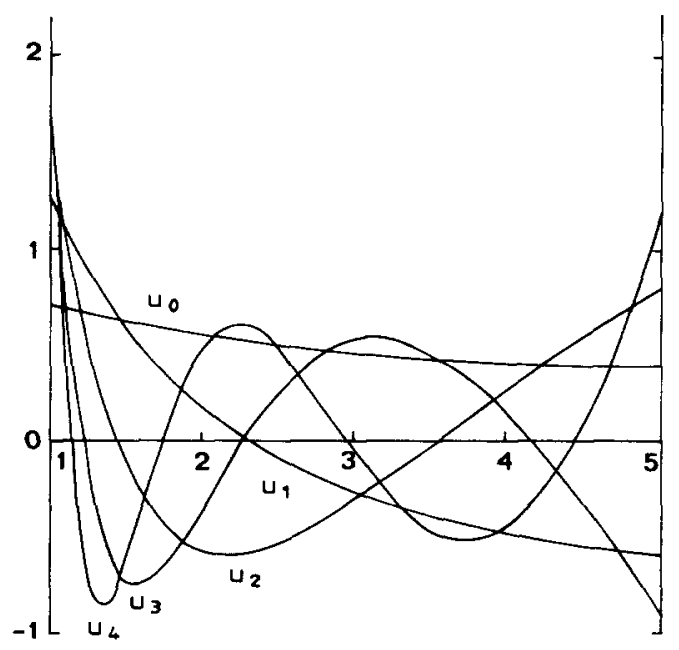

Fig. 2. Singular functions $u_{k}(t), 1 \leq t \leq \gamma, k=0,1, \ldots, 4$ in the case $\gamma=5$.

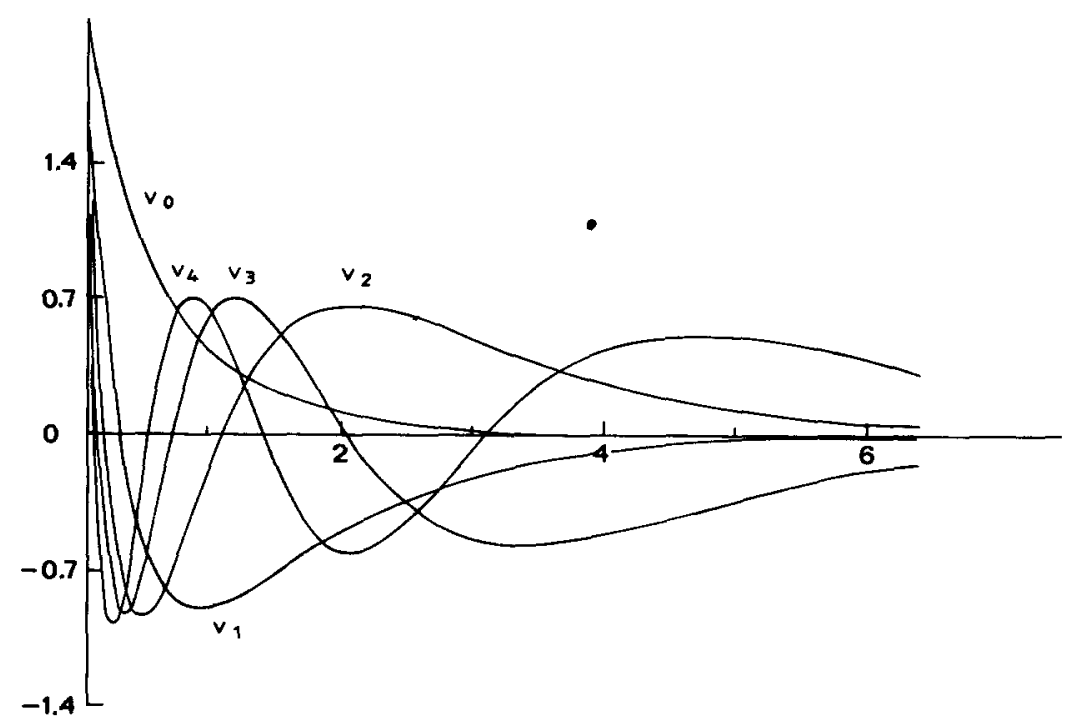

Fig. 3. Singular functions $v_{k}, k=0,1, \ldots, 4$ in the case $\gamma=5$.

For instance we have the following results (Bertero et al., 1982) in the case $\gamma=5$

$$
\alpha_{0}=0.875 \quad \alpha_{1}=0.193 \quad \alpha_{2}=0.038 \quad \alpha_{3}=0.007 \text {. }
$$

The method of optimum filtering can also be applied to equation (20) and the result is that we must keep only the singular values satisfying the condition

$$
\alpha_{k} \geq \varepsilon / E
$$

which is analogous to equation (8). We denote by $K_{s}$ the maximum value of the index of the singular values satisfying condition (21), so that $K_{s}+1$ is the number of terms we keep in equation (20) (number of degrees of freedom).

The singular functions $u_{k}(t)$ have exactly $k$ zeros in the interval $[1, \gamma]$ (Bertero and Grünbaum 1985); furthermore, as follows from numerical computations, these zeros are approximately equidistant in the variable $\ln (t)$. As a consequence we take as resolution elements the intervals between adjacent zeros of the singular function of highest order, i.e. 
$k=K_{s}$ and we define as a resolution ratio $\delta_{s}$ the quantity given by (Bertero et al., 1982)

$$
\delta_{s}=\gamma^{1 / K_{s}} \text {. }
$$

This quantity can be computed by means of numerical calculations of the singular values of $L$ and compared with the resolution ratio $\delta_{0}$ defined in the previous section. For instance in the case $\gamma=5$ we have found $\delta_{s}=1.74,1.44,1.32$ for $E / \varepsilon=10^{2}, 10^{3}, 10^{4}$. These figures, compared with the values of $\delta_{0}$ derived from equation (12), demonstrate the beneficial effect of the knowledge of the location of the solution on the resolution limits.

\section{THE CASE OF DISCRETE DATA}

In a practical problem the Laplace transform is not known for all the values of $p$ but only at a finite set of points $p_{1}, p_{2}, \ldots, p_{N}$ and therefore we have to solve the problem

$$
g_{n}=\left(L_{N} f\right)\left(p_{n}\right)=\int_{1}^{\gamma} \mathrm{e}^{-p_{n} t} f(t) \mathrm{d} t ; n=1, \ldots, N,
$$

where the $g_{n}$ are the measured values of the Laplace transform and $L_{N}$ is the operator transforming the unknown function $f(t)$ into a vector of a euclidean space. Then the adjoint operator is given by

$$
\left(L_{N}^{*} \mathbf{g}\right)(t)=\sum_{n=1}^{N} w_{n} g_{n} \mathrm{e}^{-p_{n} t},
$$

where $\mathbf{g}$ is the vector with components $g_{n}$ and the $w_{n}$ are the weights defining the scalar product in the data space (Bertero et al., 1985a).

The advantage of the method of singular function expansions is that it can also be applied to the present case. We denote by $\left\{\alpha_{N, k} ; u_{N, k}, v_{N, k}\right\}$ the singular system of $L_{N}$, i.e. the set of the solutions of the coupled equations

$$
L_{N} u_{N, k}=\alpha_{N, k} v_{N, k}, \quad L_{N}^{*} v_{N, k}=\alpha_{N, k} u_{N, k} .
$$

The computation of this system is quite easy since the operator $\hat{L}_{N}=L_{N} L_{N}^{*}$ is just a matrix $N \times N$ (it is related to the Gram matrix of the exponentials $\exp \left(-p_{n} t\right)$ (Bertero et al., 1985a)) and therefore one can reduce the solution of the problem (25) to a standard eigenvalue problem for a symmetric matrix.

Now the solution of minimal norm of problem (23) is given by an expansion similar to equation (20)

$$
f^{\dagger}(t)=\sum_{k=0}^{N-1} \frac{1}{\alpha_{N, k}}\left(\mathbf{g}, v_{N, k}\right) u_{N, k}(t)
$$

and also this solution is affected by numerical instability if $N$ is too large. Since it is possible to show, by means of numerical computations, that the singular values $\alpha_{N, k}$ can provide good approximations of the singular values $\alpha_{k}$ defined in the previous section, we conclude that the method of optimum filtering will select the same number of terms in equations (20) and (26).

We have considered two data-point distributions (Bertero et al., 1985a): a set of $N$ equidistant points, $p_{n}=c+d(n-1)$, characterized by two parameters, the position $c$ of the first point and the distance $d$ between adjacent points, and also a set of $N$ points forming a geometric progression, $p_{n}=c \Delta^{n-1}$, characterized again by two parameters, the position $c$ of the first point and the dilation factor $\Delta$ giving the ratio between adjacent data points.

The conclusion of several numerical computations, directed towards the optimum determination of the number and location of the data points, is that the points forming a geometric progression provide much better approximations of the singular values than the equidistant points. Furthermore the number of points required is much smaller in this case than in the case of equidistant points. This result justifies the choice of the integrated logarithmic sampling scheme used in some photon correlators, as we mentioned in the Introduction. 
As an example of our results we give the first three singular values for the case $\gamma=5$, obtained using five geometrically spaced data points (Bertero et al., 1985a)

$$
\alpha_{N, 0}=0.874, \quad \alpha_{N, 1}=0.191, \quad \alpha_{N, 2}=0.038 \text {. }
$$

Comparing with the values of $\alpha_{k}$, always for $\gamma=5$, given in the previous section, we find an excellent agreement.

\section{INVERSION IN WEIGHTED SPACES}

The method described in the previous sections, namely the inversion of the finite Laplace transform using optimum filtering, presents some unsatisfactory features. In practical situations the support of the unknown function is not known exactly although some estimations can be made from the experimental data since the derivatives of the Laplace transform $g(p)$ at the point $p=0$ are related to the moments of the unknown function $f(t)$. The first and second moments provide information about the 'localization' of $f(t)$ but no precise estimation of its support, i.e. of the interval where $f(t)$ is not identically zero.

A second and more serious difficulty originates from the behaviour of the singular functions $u_{k}(t)$ at the edges of the support, the interval $[1, \gamma]$. As follows from Fig. 2, these functions are quite large at $t=1$ and $t=\gamma$ and therefore they are large precisely in those regions where the unknown function is presumably small. This fact gives rise to spurious and troublesome edge effects.

A way to overcome these difficulties is to look for solutions of the Laplace transform inversion in a weighted $L^{2}$ space. In such a way we are not forced to a rigid choice of the support, we can use the information contained in the knowledge of the lower moments of the unknown function and we can force the solution to be small in those regions where it is supposed to be small.

We can formulate the problem as follows (Bertero et al., 1985b): find a solution of equations (4) and (5) satisfying the condition

$$
\int_{0}^{+\infty} \frac{f^{2}(t)}{P^{2}(t)} \mathrm{d} t<+\infty
$$

where $P(t)$ is a given profile function (for instance a gamma distribution or a lognormal distribution) whose first and second moments have been adapted to the corresponding moments of $f(t)$.

If we put $f(t)=P(t) \Phi(t)$ then the inversion of the Laplace transformation in the weighted $L^{2}$-space whose norm is given by equation (27) is equivalent to the inversion in $L^{2}(0,+\infty)$ of the following operator

$$
(K \Phi)(p)=\int_{0}^{+\infty} \mathrm{e}^{-p t} P(t) \Phi(t) \mathrm{d} t .
$$

This operator is compact [more precisely of the Hilbert-Schmidt class (Reed and Simon, $1970)]$ if the profile function $P(t)$ satisfies the following condition

$$
\int_{0}^{+\infty} \frac{P^{2}(t)}{t} \mathrm{~d} t<+\infty
$$

Then one can introduce again the singular system of $K$ and use optimum filtering for determining a stable approximate solution of the problem.

We have performed several computations, also in the case of discrete data with a uniform or geometric distribution of the data points, assuming that $P(t)$ is a gamma distribution (also called Schulz function)

$$
P(t)=\frac{\beta^{\beta}}{\Gamma(\beta)} t^{\beta-1} \mathrm{e}^{-\beta t}
$$

whose first moment is unity and whose second central moment is given by $Q=1 / \beta$. Several numerical experiments have been performed in order to relate $Q$ to the experimental polydispersity factor. The application of the method to experimental data on coagulating 


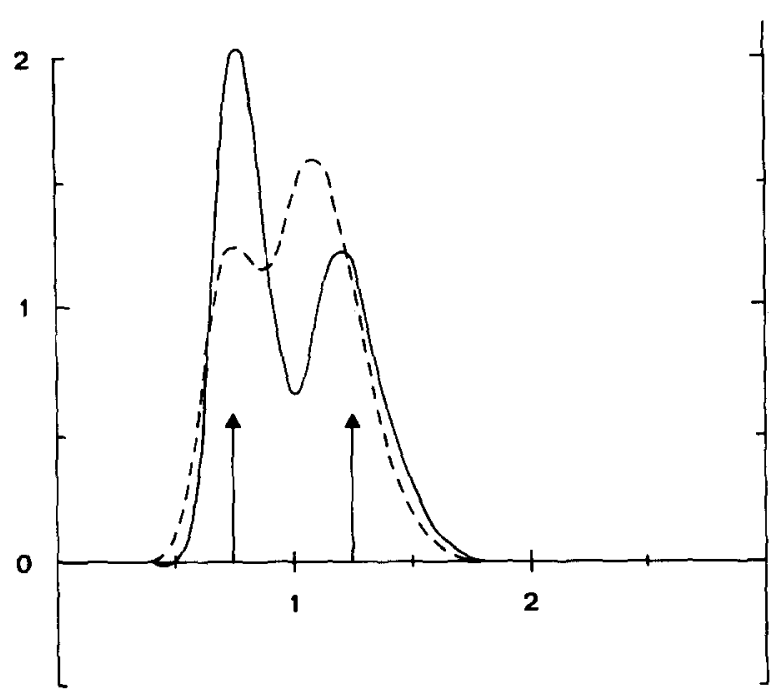

Fig. 4. Reconstruction of $f(t)=c_{1} \delta\left(t-t_{1}\right)+c_{2} \delta\left(t-t_{2}\right)$, with $c_{1}=c_{2}=0.5, t_{1}=0.75, t_{2}=1.25$ using singular functions corresponding to $\beta=16$ in equation (27), in the case of signal-to-noise ratio of the order of $10^{2}$ (broken curve) and of the order of $10^{3}$ (full curve). The arrows indicate the positions of the delta functions.

vesicle suspensions has provided satisfactory results (Bertero et al., 1985c). Here we only give an example (see Fig. 4) of a reconstruction obtained with this method. Notice that the ratio between the values of the two exponentials is $t_{2} / t_{1}=1.66$ and that it is possible to resolve these exponentials already in the case of a signal-to-noise ratio $E / \varepsilon=10^{2}$.

Acknowledgements-This work has been partly supported by NATO Grant No. 463/84, by EEC contract No. STE J-0089-3 and by Ministero della Pubblica Istruzione, Italy. C. De Mol is 'Chercheur qualifié' of the Belgian National Fund for Scientific Research.

\section{REFERENCES}

Bertero, M., Boccacci, P. and Pike, E. R. (1982) A singular value analysis of the Laplace transform inversion in the presence of noise. Proc. R. Soc. A383, 15-29.

Bertero, M., Brianzi, P. and Pike, E. R. (1985a) The effect of sampling and truncation of data on the Laplace transform inversion. Proc. R. Soc. A398, 23-44.

Bertero, M., Brianzi, P. and Pike, E. R: (1985b) Laplace transform inversion in weighted spaces. Inverse Problems 1, $1-15$.

Bertero, M., Brianzi, P., Pike, E. R., De Villiers, G., Lan, K. H. and Ostrowsky, N. (1985c) Light scattering polydispersity analysis of molecular diffusion by Laplace transform inversion in weighted spaces. J. chem. Phys. 82, 1551-1554.

Bertero, M. and Grünbaum, F. A. (1985) Commuting differential operators for the finite Laplace transform. Inverse Problems 1, 181-192.

Bertero, M., Grünbaum, F. A. and Rebolia, L. (1986) Spectral properties of a differential operator related to the inversion of the finite Laplace transform. Inverse Problems 2, 131-139.

Bertero, M., Boccacci, P., De Mol, C. and Pike, E. R. (1987a) Particle size distributions from Fraunhofer diffraction. Proceedings of the International Symposium on Optical Particle Sizing: Theory and Practice, Rouen, 12-15 May 1987.

Bertero, M., De Mol, C. and Pike, E. R. (1987b) Particle sizing by inversion of extinction data. Proceedings of the International Symposium on Optical Particle Sizing: Theory and Practice, Rouen, 12-15 May 1987.

Chowdhury, D. P., Sorensen, C. M., Taylor, T. W., Merklin, J. F. and Lester, T. W. (1984) Application of photon correlation spectroscopy to flowing Brownian motion systems. Appl. Optics. 23, 4149-4154.

Cummins, H. Z. and Pike, E. R. (Editors) (1977) Photon Correlation Spectroscopy and Velocimetry. Plenum, New York.

King, G. B., Sorensen, C. M., Lester, T. W. and Merklin, J. F. (1982) Photon correlation spectroscopy used as a particle size diagnostic in sooting flames. Appl. Optics. 20, 976-978.

McWhirter, J. G. and Pike, E. R. (1978) On the numerical inversion of the Laplace transform and similar Fredholm integral equations of the first kind. $J$. Phys. A.11, 1729-1745.

Ostrowsky, N., Sornette, D., Parker, P. and Pike, E. R. (1981) Exponential sampling method for light scattering polydispersity analysis. Optica Acta 28, 1059-1070.

Reed, M. and Simon, B. (1970) Functional Analysis (Methods of Modern Mathematical Physics, Vol. I). Academic Press, New York.

Tikhonov, A. N. and Arsenine, V. Y. (1977) Solutions of Ill-Posed Problems. Wiston/Wiley, Washington.

Titchmarsh, E. C. (1948) Introduction to the Theory of Fourier Integrals. Clarendon, Oxford. 